\title{
Influence of Lower Limb Clinical Physical Measurements of Female Athletes on Knee Motion During Continuous Jump Testing
}

\author{
Yasuharu Nagano ${ }^{*}, 1$, Mako Fukano ${ }^{2}$, Kaori Itagaki ${ }^{2}$, Sayori $\mathrm{Li}^{3}$, Shumpei Miyakawa ${ }^{4}$ and \\ Toru Fukubayashi ${ }^{1}$
}

${ }^{I}$ Faculty of Sport Sciences, Waseda University, Saitama, Japan

${ }^{2}$ Graduate School of Sport Sciences, Waseda University, Saitama, Japan

${ }^{3}$ Tsukuba Medical Center Hospital, Ibaraki, Japan

${ }^{4}$ Graduate School of Comprehensive Human Sciences, University of Tsukuba, Ibaraki, Japan

\begin{abstract}
Objectives: To assess the relationship between dynamic knee motion in female athletes during landing after jumping and lower limb clinical physical measurements, considered risk factors for anterior cruciate ligament (ACL) injury. We proposed that (1) knee valgus and flexion angles during landing are correlated with clinical physical measurements; (2) combining these measurements enables prediction of the knee valgus and flexion angles during landing.

Methods: Sixty-one female collegiate basketball athletes performed a continuous jump test; the peak knee valgus and flexion angles were measured. The Q-angle, the ranges of motion (ROMs) of hip internal rotation (IR) and external rotation (ER), as well as ankle dorsiflexion (DF), navicular drop, leg-heel alignment, and balance ability as assessed by the Star Excursion Balance Test (SEBT) were measured. Stepwise linear regression analyses were used to assess whether these factors can predict the peak knee valgus or flexion angle.

Results: Increased ROM of hip IR and navicular drop predicted $7.9 \%$ of the peak knee valgus angle variance. Increased ROMs of ankle DF and hip IR, navicular drop, and anterior balance predicted $29.0 \%$ of the peak knee flexion angle variance. The knee valgus and flexion angles during the continuous jump test were slightly correlated with clinical physical measurements.

Conclusions: Proximal and distal joint alignment and balance ability influence knee motion during landing. The relationship between knee motion during landing and these factors is weak; therefore, lower limb movement during landing is almost independent of clinical physical measurements, and knee movement should be evaluated by itself.
\end{abstract}

Keywords: Balance/stability, injury, knee, landing, motion analysis.

\section{INTRODUCTION}

The anterior cruciate ligament (ACL) is the most frequently injured knee ligament [1]. The probability of sustaining an ACL injury is 2-8 times greater for females than for males [1-4]. When examining the mechanisms and risk factors related to ACL injury, several topics are typically proposed, including joint biomechanics, neuromuscular control, and lower limb alignment. The aetiology of ACL injuries is determined not by a single decisive factor but by several factors [5].

Regarding ACL injuries that occur during sports activities, approximately $70 \%$ result from non-contact mechanisms $[2,3,6]$. Previous video analyses have revealed that most ACL injuries occur in non-contact situations during a foot strike from sudden stopping, cutting, or landing [6-8]. The knee position at the moment of injury shows a

*Address correspondence to this author at the Faculty of Sport Sciences, Waseda University, Mikajima 2-579-15, Tokorozawa, Saitama 359-1192, Japan; Tel: +81-4-2947-6853; Fax: +81-4-2947-1314;

E-mail: yasu-nagano@aoni.waseda.jp slight knee flexion and a greater valgus angle with internal or external tibial rotation [8]. Hewett et al. [9] measured 205 female athletes prospectively by using three-dimensional kinematics (joint angles) and joint loads using kinetics (joint moments) during a jump-landing task. Their data showed that female athletes with increased dynamic valgus and high abduction loads have an increased risk of ACL injury. In fact, prevention programmes for ACL injury emphasise dynamic knee motion avoiding knee valgus collapse and slight knee flexion [10-12]. Therefore, greater knee valgus and slight knee flexion during landing, stopping, and cutting is thought to be an ACL-injury risk factor.

Neuromuscular control, as measured by balance ability, is also an important factor in examining ACL-injury mechanisms. Plisky et al. [13] reported that neuromuscular control demonstrated by balance ability assessed by the Star Excursion Balance Test (SEBT) predicted lower extremity injury. The possibility exists that balance ability also affects knee motion, which influences the likelihood of ACL injury. Static lower limbs as evidenced by clinical physical measurements are also thought to be risk factors. Foot hyperpronation, as measured by the navicular drop [14-16] 
and calcaneal angle $[15,16]$, has also been examined as an ACL-injury risk factor. The Q-angle, an indicator of lower limb alignment, shows a greater mean in females than in males [17]. The ranges of motion (ROMs) of hip rotation and ankle dorsiflexion (DF) are often measured as indicators of lower limb characteristics. Because clinical physical measurements can be easily observed, these factors are useful for screening athletes at high risk of injury. Moreover, according to our hypothesis, one or some clinical physical measurements influence dynamic knee motion. Some factors can cause high-risk knee motions, such as greater knee valgus and slight knee flexion. Nevertheless, their implication in ACL injury and the relationship between dynamic knee motion and clinical physical measurements remain unknown.

The aim of this study was to assess the relationship between dynamic knee motion during landing and lower limb clinical physical measurements. In this study, the lower limb clinical physical measurements included the Q-angle, the ROMs of hip IR and ER as well as ankle DF, navicular drop, leg-heel alignment and balance ability assessed by SEBT. By understanding these relationships, we can determine whether clinical physical measurements can predict knee motion during landing. For cases in which these factors are predictive of knee motion during landing, we should consider them to improve knee motion during landing. We proposed two hypotheses: (1) knee valgus and flexion angles during landing are correlated with clinical physical measurements; (2) combining these factors enables prediction of the knee valgus and flexion angles during landing.

\section{METHODS}

\section{Study Population}

In all, 61 female collegiate basketball athletes from three college basketball teams participated. The inclusion criterion was a lack of current lower extremity injury, neuromuscular disorder or any previous history that would detract from the ability to perform a jump or balance test. We analysed both legs of each subject. The subjects' mean (SD) age, height, and weight was $19.4(1.2)$ years, $169.1(6.6) \mathrm{cm}$, and 62.8 (6.5) $\mathrm{kg}$, respectively. All subjects gave written informed consent approved by Waseda University. The study protocol was approved by the institutional review board of Waseda University.

\section{Continuous Jump Testing}

All subjects were required to perform vertical jumps five times by using both legs with maximal effort as detailed in a previous study [18]. Participants were instructed to place their hands on the lower torso, stand with feet apart at shoulder width and face a video camera to allow the proper recording of movements (Fig. 1). A research assistant first demonstrated the jumps. The subjects were verbally instructed to shorten their foot contact time to the greatest extent possible and to jump as high as they could. They performed several preparatory trials. Measurements were continued until successful trials had been accomplished for both legs, i.e. first, the subject was filmed frontally and sagittally on both sides by digital video cameras $(30 \mathrm{~Hz}$; Panasonic Inc., Japan). If a subject moved out of view of the camera or when the landing position deviated from the start position, the trial was excluded. The frontal plane camera was placed at a distance of $3.5 \mathrm{~m}$ from both feet. The sagittal plane camera was placed at a $3.5 \mathrm{~m}$ from the line formed by both lateral malleoli. Each video camera was placed at knee joint height.

For each subject, twelve $1.8 \mathrm{~cm} \times 1.8 \mathrm{~cm}$ plastic tape markers were secured to the lower limbs. The markers were placed on both the right and left anterior superior iliac spines (ASIS), both midpoints of the patellae, both midpoints of the medial and lateral malleoli on their shoes, both greater trochanters, and finally, both lateral knee joint lines. The captured images were imported into a digitizing software program (Dartfish software; Dartfish Co. Ltd., Japan). The angle formed by the lines from the marker on the ASIS to the midpoint of the patella, and from the midpoint of the patella to the midpoint of the medial and lateral malleoli was recorded as the knee valgus angle. The angle between the lines formed by the marker on the greater trochanter and the lateral knee joint line and from the lateral knee joint line to the lateral malleolus was recorded as the knee flexion angle. The average peak knee valgus and flexion angles from the second to the fourth landings were measured for analyses. The average peak knee valgus angle was corrected using a linear regression equation [18]. Because this equation was not applicable to the knee varus, the data obtained when subjects exhibited knee varus during landings were excluded.

\section{Lower Limb Clinical Physical Measurements}

\section{Balance Ability Testing}

We followed the Star Excursion Balance Test (SEBT) procedure described by Plisky et al. [13] by using a custommade device. The device consisted of a footplate and three measuring cords with an attached slider extending in the anterior, posteromedial, and posterolateral directions (Fig. 2). The participants stood on one leg on the footplate, with the most distal aspect of the great toe at the starting line. While maintaining the single-leg stance, the participants were asked to reach with the free leg to the anterior, posteromedial, and posterolateral directions in relation to the stance foot. The maximum reach distance was measured by moving the slider from the starting point of the toe of the stationary foot to the most distant point of the extended foot. The trials were discarded and repeated if the participants (1) failed to maintain a unilateral stance, (2) lifted or moved the stance foot from the grid, (3) touched down with the reach foot, or (4) failed to return the reach foot to the starting position. The participants practised three trials on each leg in each of the three reach directions before formal testing. After the practice, they conducted formal testing of three trials on each leg in each of the three reach directions. The greatest value from the three trials for each direction was used for the analysis of each reach distance, and then the process was repeated with the other leg. In addition, the greatest reach distance in each direction was combined to yield a composite reach distance for analysis of overall test performance. The lengths of both legs were measured in a standing position from the ASIS to the top portion of the medial malleolus by using a cloth tape measure, and the length data were normalised for each leg. The intraclass correlation coefficients (ICC) of this test $\left(\mathrm{ICC}_{1,1}: n=14\right.$ legs, with 


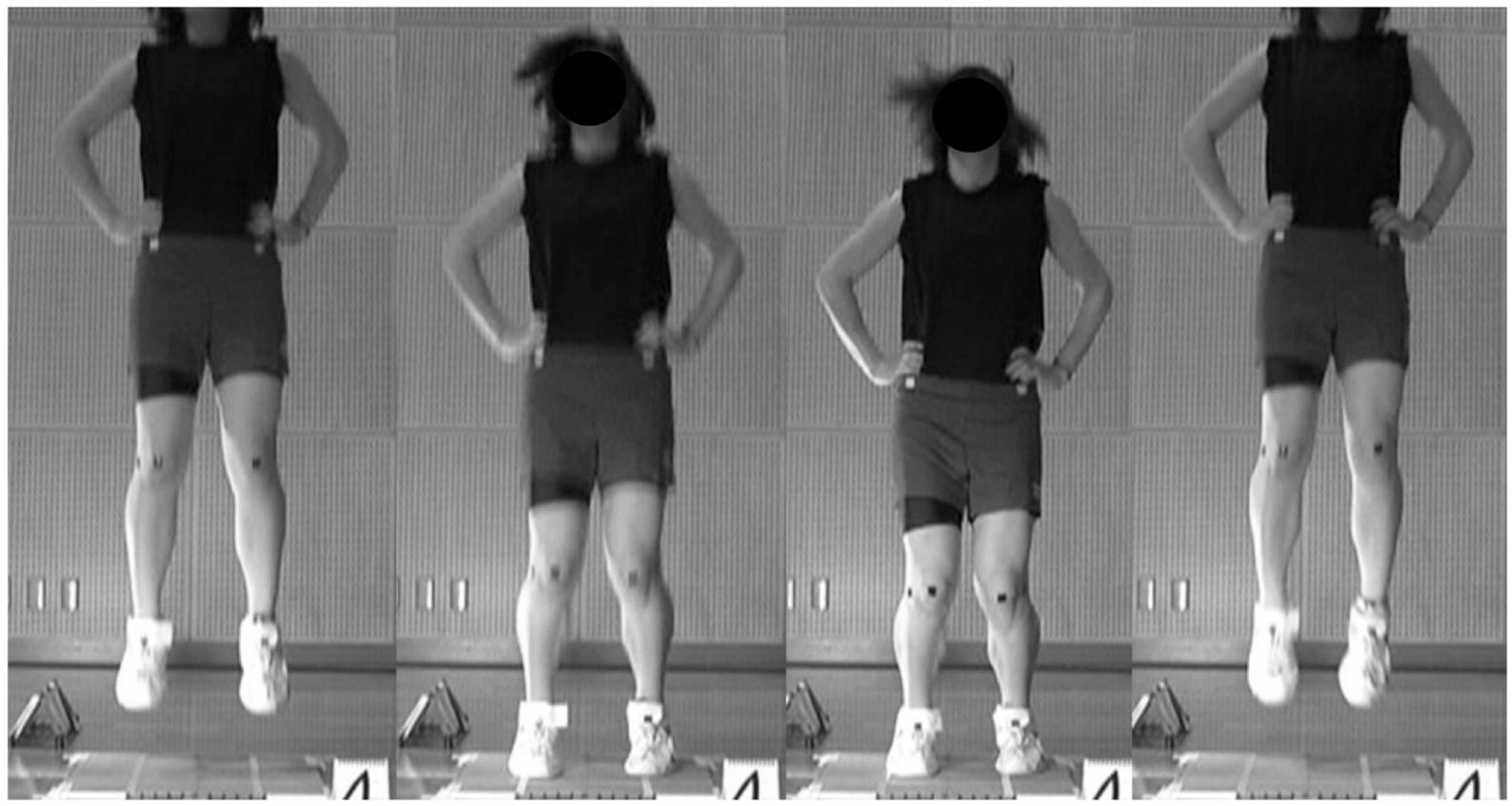

Fig. (1). Continuous jump test. All subjects performed five vertical jumps and landings using both legs with maximum effort.

testing-retesting thrice) were high: $0.78,0.76,0.71$, and 0.76 for the anterior, posteromedial, posterolateral, and composite measurements, respectively.

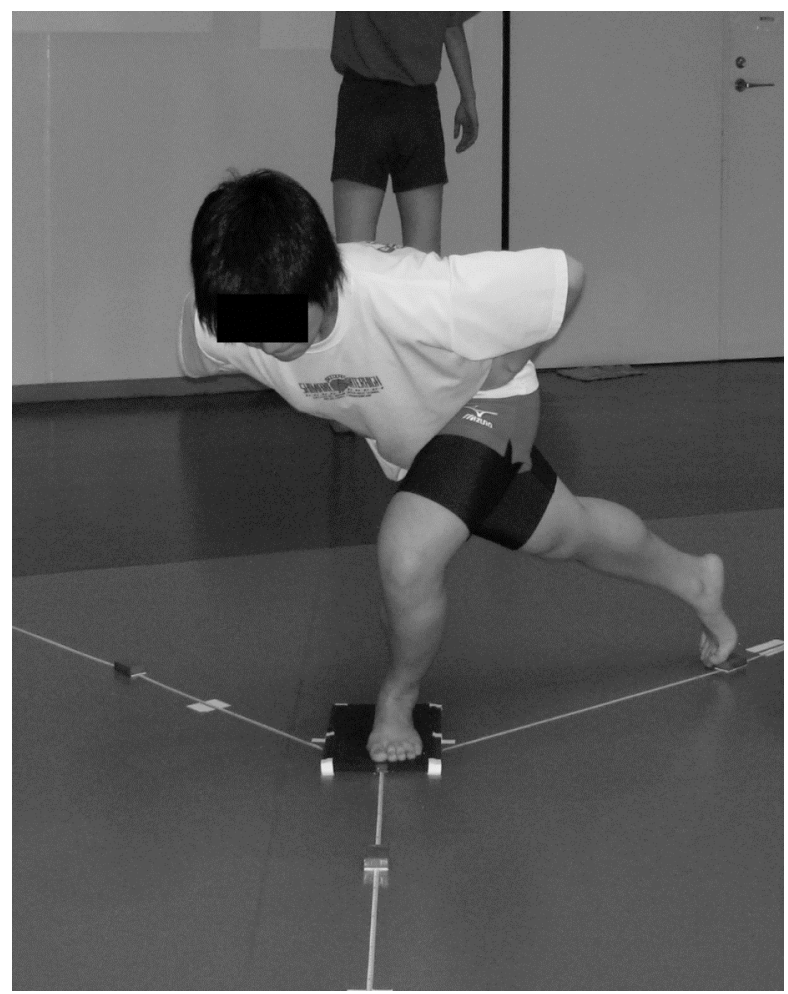

Fig. (2). Star Excursion Balance Test. The Star Excursion Balance Test procedure was performed with a device that we created, comprising of a footplate and three measuring cords with a slider spreading in the anterior, posteromedial and posterolateral directions. While maintaining a single-leg standing position, the participant was asked to extend the free lower limb in the anterior, posteromedial, and posterolateral directions in relation to the fixed foot.

\section{Q-Angle}

The Q-angle was measured in the frontal plane as the angle formed between the intersecting lines from the top of the ASIS to the centre of the patella and from the centre of the patella to the centre of the tibial tuberosity in a standing position [19] by using a protractor with an attached cord to achieve accurate alignment with the ASIS.

\section{ROMs of Hip IR and ER}

The hip was rotated passively until immediately before the pelvis rotated. Using an inclinometre (Multi Level A300; Shinwa Sokutei K.K., Japan), the angle created by the tibia in the prone position with the knee flexed at 90 degrees and vertical was measured. In addition, the midpoint of hip rotation (MPR) was calculated using the following formula:

$M P R=(I R-E R) / 2\left(^{\circ}\right)$

We considered that a positive MPR value indicated anterior femoral torsion.

\section{ROM of Ankle DF}

The ROM of ankle DF was measured using a modification of the standing bent knee measurement described by Denegar et al. [20]. A Velcro strap was secured immediately above one-third of the talocrural joint and around the subject's lower leg, with the inclinometre adhered to the strap and facing laterally. To zero the inclinometre, the subject was asked to stand relaxed with feet apart at shoulder width. The foot of the limb being tested was aligned so that it was parallel to the long axis of the lower leg. The subject was instructed to perform a single limb squat slowly by flexing the hip and knee joints. To maintain balance, the subject was instructed to touch the floor with the toe of the opposite foot while holding onto a post, as opposed to the previously reported method [20]. The measurement was taken once the subject's heel began to rise off the floor. The 
ICC of this test $\left(\mathrm{ICC}_{1,1}: n=14\right.$ legs, with testing-retesting thrice) was substantial: 0.83 .

\section{Navicular Drop Test}

The navicular drop represents a composite measure of foot pronation [21]. The procedure was modified from Brody's test [22]. The navicular drop was measured as the distance between the navicular tuberosity and the floor during a quiet sitting stance. The subtalar joint was placed in a neutral position, in which the medial and lateral aspects of the talar head were equally palpable on both sides, without loading the foot and with a relaxed bilateral stance. The difference in navicular height between sitting and standing was recorded in millimetres by using a digital calliper with an index card [14] or straight edge ruler, as reported in earlier studies [23]. The ICC of this test $\left(\mathrm{ICC}_{1,1}: n=8\right.$ legs, with testing-retesting thrice) was also substantial: 0.75.

\section{Leg-Heel Alignment}

The leg-heel alignment represents pronation of the rear foot. The leg-heel alignment was measured by using a modification of the technique described by WoodfordRogers et al. [16]. The lower third of the leg was bisected from the musculotendinous junction of the triceps surae to the Achilles tendon. The medial and lateral tubercles of the calcaneus were palpated, and the calcaneus was bisected. With the subject in the subtalar joint neutral position (defined in the same manner as in the navicular drop testing), a goniometre was used to estimate the angle between the lines joining the bisections of the leg and the calcaneus in a full-weight bilateral standing stance. The ICC of this test $\left(\mathrm{ICC}_{1,1}: n=8\right.$ legs, with testing-retesting thrice) was almost perfect: 0.92 .

\section{Statistical Analysis}

By setting the effect size correlation coefficient $(r)$ at 0.30 and the statistical power at 0.80 , a sample size calculation revealed that data for 85 legs were necessary to yield sufficient inferential power [24]. The association between the peak knee valgus and flexion angles, and the clinical physical measurements (anterior, posteromedial, posterolateral and composite balance ability, Q-angle, ROMs of hip IR and ER as well as ankle DF, navicular drop, and leg-heel alignment) were analysed using the Pearson product-moment correlation coefficients. For this study, we set the $r$ value at $0.7 \leq|r|$ for strong, at $0.3 \leq|r|<0.7$ for moderate, and at $|r|<0.3$ for weak correlations, because the coefficient of determination $\left(r^{2}\right)$ is higher than 0.5 for strong correlations and lower than 0.1 for weak correlations. In addition, stepwise linear regression analysis was used to examine whether clinical physical measurements were predictive of the peak knee valgus or flexion angles during the continuous jump test. Statistical significance was inferred for $p<0.05$.

\section{RESULTS}

In the knee valgus measurement during the continuous jump testing, of the 61 subjects tested (122 legs), two displayed a varus knee angle in both legs (4 legs) and four displayed a varus knee angle in one leg (4 legs) were excluded. Therefore, 59 subjects and 114 legs were analysed in this study. The average peak knee valgus angle was $5.2 \pm$ $(2.2)^{\circ}$; the average peak knee flexion angle was $47.2(7.7)^{\circ}$. Table 1 presents the mean ( $\pm \mathrm{SD})$ and $r$ values of the lower limb characteristics and balance ability with respect to the knee valgus and flexion angles. Weak correlation was found between the peak knee valgus angle and ROM of hip IR ( $r=$ $0.20)$ as well as between the peak knee valgus angle and navicular drop $(\mathrm{r}=0.19)$. Moderate correlation was found between the peak knee flexion angle and ROM of ankle DF $(\mathrm{r}=0.42)$ as well as the peak knee flexion angle and anterior balance $(r=0.35)$. Weak correlations were found between the peak knee flexion angle and ROM of hip IR $(r=0.25)$, MPR $(r=0.21)$, navicular drop $(r=0.29)$, and leg-heel angle $(\mathrm{r}=0.19)$.

Table 1. Mean ( \pm SD) of Lower Limb Alignment $\left({ }^{\circ}\right.$ or mm) and Balance Abilities (\% Leg Length), and Correlation Values for their Association with Knee Valgus and Knee Flexion Angles

\begin{tabular}{|c|c|c|c|}
\hline & $\begin{array}{c}\text { Mean } \\
( \pm \text { SD) }\end{array}$ & $\begin{array}{c}\text { Knee Valgus } \\
\text { Angle }(\boldsymbol{r})\end{array}$ & $\begin{array}{c}\text { Knee Flex } \\
\text { Angle }(\boldsymbol{r})\end{array}$ \\
\hline \hline Q-angle & $20.1(4.6)$ & -0.05 & -0.06 \\
\hline ROM of hip IR & $49.9(10.5)$ & $0.20^{*}$ & $0.25^{* *}$ \\
\hline ROM of hip ER & $33.5(8.4)$ & -0.03 & -0.05 \\
\hline MPR & $8.2(7.4)$ & 0.16 & $0.21^{*}$ \\
\hline ROM of ankle DF & $34.5(8.7)$ & 0.06 & $0.42^{* *}$ \\
\hline ND & $5.7(3.7)$ & $0.19^{*}$ & $0.29^{* *}$ \\
\hline Leg-heel angle & $5.2(4.5)$ & 0.05 & $0.19^{*}$ \\
\hline Anterior balance & $72.6(5.7)$ & 0.06 & $0.35^{* *}$ \\
\hline Posteromedial balance & $110.1(7.2)$ & 0.07 & 0.08 \\
\hline Posterolateral balance & $108.1(9.2)$ & 0.04 & 0.03 \\
\hline Composite balance & $96.9(6.1)$ & 0.07 & 0.16 \\
\hline
\end{tabular}

ROM of hip IR, range of motion of hip internal rotation; ROM of hip ER, range of motion of hip external rotation; ROM of ankle DF, range of motion of ankle dorsiflexion; MPR, midpoint of hip rotation; ND, navicular drop. $* p<0.05 ; * * p<0.01$.

Table 2 presents the results of the stepwise linear regression analysis for the peak knee valgus angle. From $r^{2}$, increased ROM of hip IR and navicular drop predicted $7.9 \%$ of the variance in the peak knee valgus angle $(p<0.01)$. Table 3 displays the results of the stepwise linear regression analysis for the peak knee flexion angle. As inferred from $r^{2}$, increased ROMs of the ankle DF and hip IR, the navicular drop, and the anterior balance together predicted $29.0 \%$ of the variance in the peak knee flexion angle $(p<0.01)$.

\section{DISCUSSION}

In this study, we examined the correlation between dynamic knee motion during landing and clinical physical measurements. We sought to determine whether clinical physical measurements were useful in predicting knee motion during landing. We hypothesised that the knee valgus and flexion angles during continuous jump testing correlated with clinical physical measurements, and that combining these factors allows for the prediction of knee valgus and flexion angles during landing. 
Table 2. Results of a Stepwise Regression Model for the Peak Knee Valgus Angle

\begin{tabular}{|c|c|c|c|c|}
\hline Predictor Variables & $\mathbf{r}^{2}$ & Adj. $\mathbf{r}^{2}$ & SE of Est & Equation \\
\hline \hline ROM of hip IR & 0.042 & 0.033 & 2.195 & Knee valgus angle $=0.043$ (ROM of hip IR) +3.042 \\
\hline ROM of hip IR ND & 0.079 & 0.063 & 2.162 & Knee valgus angle $=0.044$ (ROM of IR) +0.116 (ND) +2.369 \\
\hline
\end{tabular}

ROM of hip IR; range of motion of hip internal rotation; ND, navicular drop.

Table 3. Results of a Stepwise Regression Model for the Peak Knee Flexion Angle

\begin{tabular}{|c|c|c|c|c|}
\hline Predictor Variables & $\mathbf{r}^{2}$ & Adj. $\mathbf{r}^{2}$ & SE of Est & Equation \\
\hline \hline ROM of ankle DF & 0.180 & 0.172 & 7.017 & Knee flexion angle $=0.375($ ROM of ankle DF) +34.228 \\
\hline ROM of ankle DF ND & 0.234 & 0.220 & 6.812 & Knee flexion angle $=0.347$ (ROM of ankle DF) $+0.486(\mathrm{ND})+32.453$ \\
\hline $\begin{array}{c}\text { ROM of ankle } \\
\text { DF ND AT }\end{array}$ & 0.277 & 0.257 & 6.648 & Knee flexion angle $=0.282($ ROM of ankle DF) $+0.456(\mathrm{ND})+0.300(\mathrm{AT})+13.056$ \\
\hline $\begin{array}{c}\text { ROM of ankle DF } \\
\text { ND AT ROM of hip IR }\end{array}$ & 0.290 & 0.264 & 6.618 & $\begin{array}{c}\text { Knee flexion angle }=0.261(\mathrm{ROM} \text { of ankle DF) }+ \\
0.471(\mathrm{ND})+0.264(\mathrm{AT})+0.089(\mathrm{ROM} \text { of hip IR) }+11.889\end{array}$ \\
\hline
\end{tabular}

ROM of ankle DF, range of motion of ankle dorsiflexion; ND, navicular drop; AT, anterior balance; ROM of hip IR, range of motion of hip internal rotation.

A trend towards greater knee valgus has been observed in ACL injury [6-8]. In particular, female athletes with increased dynamic valgus and high abduction loads are at increased risk for ACL injury [9]. Therefore, the knee valgus angle is thought to be an indicator of the risk of ACL injury. We found that greater ROM of hip IR and greater navicular drop contributed slightly to greater knee valgus during landing. ROM of hip IR is thought to reflect greater hip IR during landing and to induce inward collapse of the knee. Navicular drop represents composite foot pronation and function of the medial longitudinal arch. Greater pronation and dysfunction of the medial longitudinal arch is thought to incline the tibia inwards and induce inward collapse of the knee. The results of this study suggested that frontal knee motion is influenced by the proximal and distal joint alignments and characteristics.

A small amount of knee flexion is often observed in ACL injury [6-8]; for example, Teitz [7] reported that the angle of knee flexion at the time of injury was less than $30^{\circ}$. Additionally, in a case of slight knee flexion $\left(<30^{\circ}\right)$, contraction of the quadriceps was observed to strain the ACL [25-27], and therefore, it is also considered a risk factor for ACL injury. We found that greater ROM of ankle DF, greater navicular drop, greater anterior balance, and greater ROM of hip IR may relate to greater knee flexion during landing. The limited ROM of ankle DF is thought to limit forward tilting of the shank and to restrict knee flexion. Limitation of foot pronation, represented by navicular drop, and ROM of hip IR can restrict the absorption of the landing force, not only in the knee joint but also throughout the entire lower limb. Normal foot pronation and ROM of hip IR would allow effective absorption of the landing force and knee flexion. On the other hand, our results indicate that excessive foot pronation and ROM of hip IR are related to greater knee valgus, and therefore, are not desirable considering the overall knee motion during landing. The relationship between poor anterior balance and lower limb injury has been reported recently [13]; the possibility exists that poor anterior balance and induced knee motion increase lower limb injury.
Although our results indicated that lower limb alignment can predict knee motion statistically, the factors used in this study predicted only $7.9 \%$ and $29.0 \%$ of the variance. In other studies, the relationship between a commonly used functional movement assessment and clinical physical measurements was low or not significant. Sigward et al. [28] examined the association between frontal plane knee excursion and clinical measures of hip strength and ROMs of the ankle and hip; they reported a small relationship between the ROMs and frontal plane knee excursion. Mizner et al. [29] reported that landing kinematics are independent of hip muscle strength. Considering the results of these previous studies and our study, although knee motion during landing is partially attributable to lower limb clinical physical measurements, the lower limb movement during landing, especially in knee valgus, is almost independent of clinical physical measurements, and it is necessary to evaluate the movement itself.

Our study has some limitations. First, we adopted twodimensional methods to measure knee motion during landing. Although two-dimensional knee valgus and threedimensional knee abduction had a moderate correlation [18], accurate analysis of knee abduction during landing should be conducted three-dimensionally; alternatively, twodimensional analysis could be conducted more easily for large populations. Second, the results are applicable to the relationship between knee motion during landing and lower limb clinical physical measurements. However, a causal relation between ACL injury and lower limb clinical physical measurements remains elusive. Third, we measured knee motion during peak knee flexion, but because the camera frequency was low we could not distinguish the time of toe contact. Knee motion analysis using high-frequency cameras just after ground contact will be necessary in the future.

\section{CONCLUSIONS}

We examined the correlation between dynamic knee motion during landing and lower limb clinical physical 
measurements. In addition, we investigated whether clinical physical measurements can predict knee motion during landing. The results showed that the knee valgus and flexion angles during continuous jump testing are slightly correlated with clinical physical measurements. Although combining these factors can partially explain knee valgus and the flexion angles during landing, lower limb movement during landing is almost independent of clinical physical measurements.

\section{ACKNOWLEDGEMENT}

This work was supported by a Grant-in-Aid for Scientific Research (B18300219).

\section{REFERENCES}

[1] Miyasaka KC, Daniel DM, Stone ML. The incidence of knee ligament injuries in the general population. Am J Knee Surg 1991; 4(1): 3-8.

[2] Agel J, Arendt EA, Bershadsky B. Anterior cruciate ligament injury in national collegiate athletic association basketball and soccer: a 13-year review. Am J Sports Med 2005; 33(4): 524-30.

[3] Griffin LY, Agel J, Albohm MJ, et al. Noncontact anterior cruciate ligament injuries: risk factors and prevention strategies. J Am Acad Orthop Surg 2000; 8(3): 141-50.

[4] Myklebust G, Maehlum S, Holm I, Bahr R. A prospective cohort study of anterior cruciate ligament injuries in elite Norwegian team handball. Scand J Med Sci Sports 1998; 8(3): 149-53.

[5] Huston LJ, Greenfield ML, Wojtys EM. Anterior cruciate ligament injuries in the female athlete. Potential risk factors. Clin Orthop 2000; (372): 50-63.

[6] Boden BP, Dean GS, Feagin JA, Jr., Garrett WE, Jr. Mechanisms of anterior cruciate ligament injury. Orthopedics 2000; 23(6): 5738 .

[7] Teitz CC. Video analysis of ACL injuries. In: Griffin LY, Ed. Prevention of Noncontact ACL Injuries. Rosemont, IL: American Academy of Orthopaedic Surgeons 2001; pp. 87-92.

[8] Olsen OE, Myklebust G, Engebretsen L, Bahr R. Injury mechanisms for anterior cruciate ligament injuries in team handball: a systematic video analysis. Am J Sports Med 2004; 32(4): 1002-12.

[9] Hewett TE, Myer GD, Ford KR, et al. Biomechanical measures of neuromuscular control and valgus loading of the knee predict anterior cruciate ligament injury risk in female athletes. Am J Sports Med 2005; 33(4): 492-501.

[10] Hewett TE, Lindenfeld TN, Riccobene JV, Noyes FR. The effect of neuromuscular training on the incidence of knee injury in female athletes. A prospective study. Am J Sports Med 1999; 27(6): 699706.

[11] Myklebust G, Engebretsen L, Braekken IH, et al. Prevention of anterior cruciate ligament injuries in female team handball players: a prospective intervention study over three seasons. Clin J Sport Med 2003; 13(2): 71-8
[12] Mandelbaum BR, Silvers HJ, Watanabe DS, et al. Effectiveness of a neuromuscular and proprioceptive training program in preventing anterior cruciate ligament injuries in female athletes: 2-year followup. Am J Sports Med 2005; 33(7): 1003-10.

[13] Plisky PJ, Rauh MJ, Kaminski TW, Underwood FB. Star Excursion Balance Test as a predictor of lower extremity injury in high school basketball players. J Orthop Sports Phys Ther 2006; 36(12): 911-9.

[14] Beckett ME. Incidence of hyperpronation in the ACL injured knee: A clinical perspective. J Athl Train 1992; 27: 58-62.

[15] Smith J, Szczerba JE, Arnold BL, Perrin DH, Martin DE. Role of hyperpronation as a possible risk factor for anterior cruciate ligament injuries. J Athl Train 1997; 32(1): 25-8.

[16] Woodford-Rogers B, Cyphert L, Denegar CR. Risk factors for anterior cruciate ligament injury in high school and college athletes. J Athl Train 1994; 29(4): 343-6.

[17] Shultz SJ, Nguyen AD, Schmitz RJ. Differences in lower extremity anatomical and postural characteristics in males and females between maturation groups. J Orthop Sports Phys Ther 2008; 38(3): 137-49.

[18] Nagano Y, Sakagami M, Ida H, Akai M, Fukubayashi T. Statistica modelling of knee valgus during a continuous jump test. Sports Biomech/Int Soc Biomech Sports 2008; 7(3): 342-50.

[19] Livingston LA. The quadriceps angle: a review of the literature. J Orthop Sports Phys Ther 1998; 28(2): 105-9.

[20] Denegar CR, Hertel J, Fonseca J. The effect of lateral ankle sprain on dorsiflexion range of motion, posterior talar glide, and joint laxity. J Orthop Sports Phys Ther 2002; 32(4): 166-73.

[21] Mueller MJ, Host JV, Norton BJ. Navicular drop as a composite measure of excessive pronation. J Am Podiatr Med Assoc 1993 83(4): 198-202.

[22] Brody DM. Techniques in the evaluation and treatment of the injured runner. Orthop Clin North Am 1982; 13(3): 541-58.

[23] Nguyen AD, Shultz SJ. Sex differences in clinical measures of lower extremity alignment. J Orthop Sports Phys Ther 2007; 37(7): 389-98.

[24] Hulley SB, Cummings SR, Browner WS, et al. Designing Clinical Research: An Epidemiologic Approach. $2^{\text {nd }}$ ed. Philadelphia: Williams \& Wilkins 2001.

[25] Arms SW, Pope MH, Johnson RJ, et al. The biomechanics of anterior cruciate ligament rehabilitation and reconstruction. Am J Sports Med 1984; 12(1): 8-18.

[26] Renstrom P, Arms SW, Stanwyck TS, Johnson RJ, Pope MH. Strain within the anterior cruciate ligament during hamstring and quadriceps activity. Am J Sports Med 1986; 14(1): 83-7.

[27] Beynnon BD, Fleming BC, Johnson RJ, et al. Anterior cruciate ligament strain behavior during rehabilitation exercises in vivo. Am J Sports Med 1995; 23(1): 24-34.

[28] Sigward SM, Ota S, Powers CM. Predictors of frontal plane knee excursion during a drop land in young female soccer players. J Orthop Sports Phys Ther 2008; 38(11): 661-7.

[29] Mizner RL, Kawaguchi JK, Chmielewski TL. Muscle strength in the lower extremity does not predict postinstruction improvements in the landing patterns of female athletes. J Orthop Sports Phys Ther 2008; 38(6): 353-61. 